\title{
The empirical dependence of radiation-induced charge neutralization on negative bias in dosimeters based on the metal-oxide-semiconductor field-effect transistor
}

\author{
Chris Benson, ${ }^{\text {a) }}$ Abdulrahman Albadri, ${ }^{\text {b) }}$ and Malcolm J. Joyce ${ }^{\text {c) }}$ \\ Engineering Department, Lancaster University, Bailrigg, Lancaster, Lancashire LA1 4YR, United Kingdom \\ Robert A. Price \\ Department of Radiography, School of Allied Health Sciences, City University, Northampton Square, \\ London EC1V OHB, United Kingdom
}

(Received 6 January 2006; accepted 25 May 2006; published online 22 August 2006)

\begin{abstract}
The dependence of radiation-induced charge neutralization (RICN) has been studied in metal-oxide-semiconductor field-effect transistor (MOSFET) dosimeters. These devices were first exposed to $\mathrm{x}$ rays under positive bias and then to further dose increments at a selection of reverse bias levels. A nonlinear empirical trend has been established that is consistent with that identified in the data obtained in this work. Estimates for the reverse bias level corresponding to the maximum rate of RICN have been extracted from the data. These optimum bias levels appear to be independent of the level of initial absorbed dose under positive bias. The established models for threshold voltage change have been considered and indicate a related nonlinear trend for neutralization cross section $\sigma_{N}$ as a function of oxide field. These data are discussed in the context of dose measurement with MOSFETs and within the framework of statistical mechanics associated with neutral traps and their field dependence. () 2006 American Institute of Physics.
\end{abstract}

[DOI: $10.1063 / 1.2259814]$

\section{INTRODUCTION}

The variation in threshold voltage of metal-oxidesemiconductor (MOS) devices under switched-bias conditions is primarily due to the buildup and neutralization of oxide-trapped charge. For the case where the latter has been stimulated by incident radiation, the phenomenon is often termed radiation-induced charge neutralization (RICN). In the context of qualifying commercial integrated circuits (ICs) for industrial applications, especially in aerospace, the variation in threshold voltage is often referred to as a parasitic field-oxide transistor shift. Further exposure of a device biased "off," or indeed with a negative gate potential, acts to reduce the threshold voltage shift. This decreases the leakage current significantly and thereby elevates the failure dose of a dynamically biased part. Therefore, a thorough understanding of the dynamics of RICN is important where MOS devices are subject to a combination of switched bias and radiation exposure.

MOS structures are also being used increasingly for radiation dose measurement. This is principally as metaloxide-semiconductor field-effect transistor (MOSFET) dosimeters for applications in space and for quality assurance in medical radiotherapy. RICN poses a risk in these scenarios because inadvertent reverse biasing of these devices will reverse and undermine the cumulative dose response of the device. Recovering the dose response analytically, to offset

\footnotetext{
${ }^{\text {a) }}$ Present address: Appleyard Lees, Halifax Office, 15 Clare Road, Halifax HX1 2HY, UK.

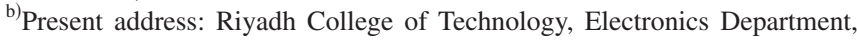
P.O. Box 42826, Riyadh 11551, Saudi Arabia.

${ }^{c)}$ Electronic mail: m.joyce@lancaster.ac.uk
}

the effect of this phenomenon, is at best difficult and will often completely undermine the validity of the response.

In contrast to the MOS devices that have been the focus of studies into RICN to date, the MOSFET dosimeter is manufactured with a thick gate oxide, in order to increase its sensitivity to ionizing radiation. Dosimeters based on the $p$-MOS field-effect transistor ${ }^{1}$ are often referred to as MOSFET dosimeters, radiation sensitive field-effect transistor (RADFETs), ${ }^{2}$ or space-charge dosimeters. They have been employed in the measurement of radiation dose in a variety of applications, including those environments associated with particle accelerators and space-bourne satellites. Perhaps the greatest potential for these devices is in the medical sector associated with dose measurement in diagnostic or therapeutic radiobiology.

There are several commercial variants and systems currently available ${ }^{3}$ for characterization and quality assurance in external beam radiotherapy. ${ }^{4}$ The main advantages of the MOSFET dosimeter are that it is small, low power, comparatively low cost, physically robust, and solid state. The MOSFET dosimeter provides an integrated response and does not require a power supply during irradiation, although improved sensitivity and reduced uncertainty can be afforded under a degree of positive gate bias.

The design of the MOSFET dosimeter has been optimized to yield a response that is independent of dose rate ${ }^{5}$ for low dose-rate applications in space. More recently, the MOSFET dosimeter has found numerous applications in nuclear medicine, ${ }^{6-9}$ for example, in mammography, ${ }_{10}^{10}$ diagnostic radiology, ${ }^{11}$ radiotherapy beam profiling, ${ }^{12}$ and intensity-modulated radiotherapy. ${ }^{13}$ The angular response has been the focus of several recent studies. ${ }^{14,15}$ Recently, we 
have explored MOSFET applications in neutron dosimetry, ${ }^{16}$ the statistical uncertainty in their response to $\mathrm{x}$ rays,${ }^{17}$ and the development of a linear MOSFET array for intracavatary in vivo dosimetry. ${ }^{18}$

The process by which the MOSFET responds to radiation is well understood. Incident ionizing radiation, typically photons, results in the liberation of electron-hole pairs. An electric field is established across the oxide, $E_{\mathrm{ox}}$, either resulting merely from the contact potential of the disparate carrier densities of the neighboring regions or enhanced by applied positive bias, and this causes the carriers to separate and drift. The electrons are swept out of the active region, while the holes are drawn to the interface with the silicon dioxide, where they become trapped. Here the holes act to reduce the depletion region of the device; a phenomenon which can be identified and studied in several ways using electrical characterization tools or impedance measurement. The change in threshold voltage $V_{t}$ can be decomposed into that due to positive oxide-trapped charge $\Delta V_{\text {ot }}$ and negatively-charged interface states $\Delta V_{\text {it }}$. While well understood, the buildup of oxide-trapped charge is a complex, nonlinear process that is dependent on the local field in the oxide, which is itself modulated by the trapped hole population.

The accepted physical model for RICN was devised by Fleetwood. ${ }^{19}$ Under positive bias, the process, as described above, results in holes trapped at the $\mathrm{Si} / \mathrm{SiO}_{2}$ interface. When the bias is switched off, $E_{\mathrm{ox}}$ is induced by the trapped positive charge and is in the opposite direction; indeed this can be exacerbated by providing negative bias to the gate during subsequent irradiation. With the field reversed, some of the trapped holes at the $\mathrm{Si} / \mathrm{SiO}_{2}$ interface are swept to the gate. However, many remain trapped. Electron-hole pairs liberated by the incident radiation are separated, but their direction of travel is reversed in comparison with the corresponding phenomenon under positive bias. The electrons migrate to the interface where they can be trapped nearby or recombine with the trapped holes. The effect of the radiation on the device's electrical parameters established under positive bias is thus neutralized.

The objective of the research reported in this paper was to explore and characterize the dependence of RICN with applied reverse bias. This measurement has been performed on devices exposed to two primary dose levels under forward bias. The optimum bias level for RICN has been estimated for each primary dose in both cases. The dependence of RICN with reverse bias has been compared with two empirical mathematical models and the most consistent is determined. The field dependence of RICN beyond the optimum bias indicates a dependence that is reduced in comparison with the known dependence of the recombination cross section $\sigma_{r}$ with electric field in the oxide. This observation may be due to a combination of compensation and recombination. The field dependence of cross section for RICN $\sigma_{N}$ represents the dependence of both phenomena.

\section{EXPERIMENTAL DETAILS}

The MOSFET devices used in this work were all supplied by the Tyndall Institute, Cork, Ireland. A photograph of

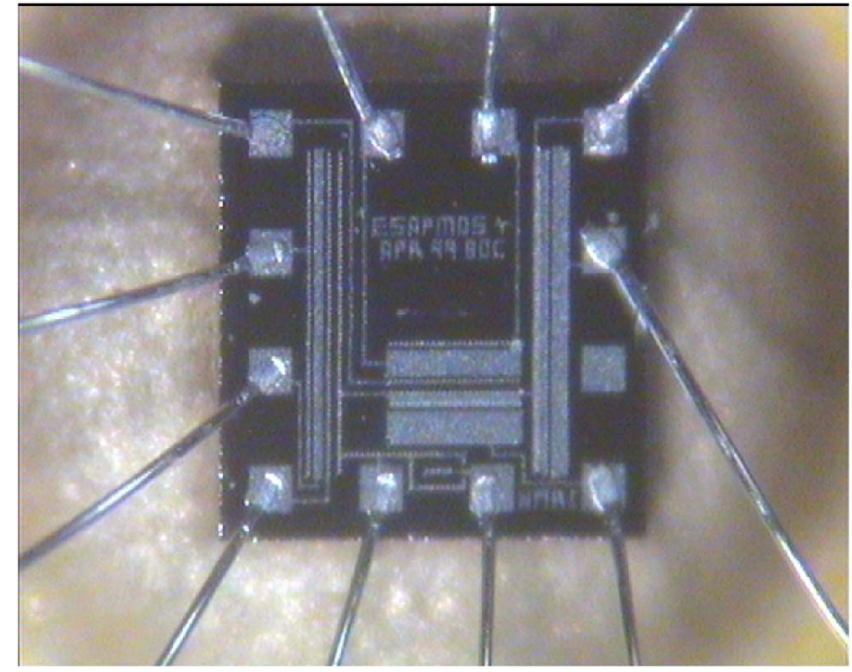

FIG. 1. Photograph of a MOSFET dosimeter die.

an example MOSFET die is given in Fig. 1. The MOSFET device die is $1 \mathrm{~mm}^{2}$ and comprises of two $300 / 50 \mu \mathrm{m}$ (width/length) devices and two 690/15 $\mu \mathrm{m}$ devices. The oxide thickness of all devices used in this study was $400 \mathrm{~nm}$. This gives each device a natural threshold voltage of between 7 and $8 \mathrm{~V}$; however, the devices used in this study have undergone an implant of boron to reduce the threshold voltage to around $0.5-2 \mathrm{~V}$. This provides a greater dynamic range for the radiation-induced threshold voltage response. ${ }^{20}$

A measurement of the threshold voltage of a MOSFET device was made with a reader circuit, as shown in Fig. 2. This arrangement measures the gate-source voltage required to conduct a fixed channel current. In this research a channel current of $10 \mu \mathrm{A}$ has been used for this purpose. The setup used in this study to measure the threshold voltage comprises an IBM personal computer (PC) housing a Hewlett Packard general purpose interface bus (GPIB) controller card, which is connected to a Keithley Instruments 2400 sourcemeter. The PC provides control over the sourcemeter through the GPIB; the sourcemeter provides electrical current sourcing and voltage measurement. The sourcemeter is configured in software to provide the $10 \mu \mathrm{A}$ channel current through a MOSFET device connected in a two-terminal reader circuit configuration, while simultaneously allowing the measure-

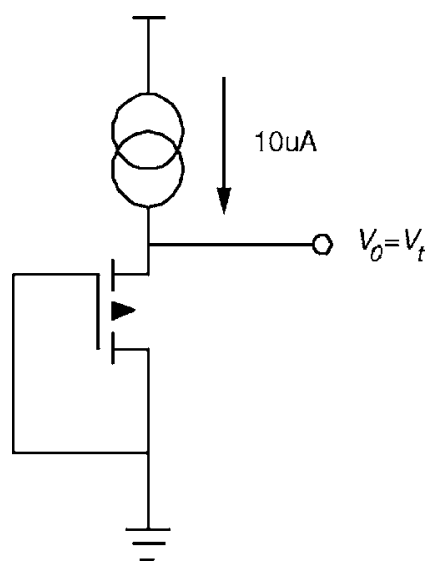

FIG. 2. The MOSFET reader circuit used in this research. 
ment of the voltage across its terminals. When the sourcemeter is connected over GPIB, this system provides an accuracy of $\pm 1 \mu \mathrm{V}$ (one standard deviation from the mean). The software used to control the sourcemeter over the GPIB was written in $\mathrm{C}$ using MICROSOFT VISUAL $\mathrm{C}++$. The threshold voltage was measured $10 \mathrm{~s}$ after the initial turn on of supply current to the MOSFET device. This provides a more stable measurement of the threshold voltage since the short-term drift due to border traps ${ }^{21}$ is reduced. ${ }^{22}$

The MOSFET dosimeters have been irradiated within a polymethylmethacrylate (PMMA) phantom to ensure charge equilibrium in the vicinity of the MOSFET and uniform radiation dose. The dimensions of the phantom were 300 $\times 300 \times 200 \mathrm{~mm}$. The PMMA phantom was placed upon a small (approximately $10 \mathrm{~cm}$ ) stack of fiberboard in order to allow sufficient depth of material below the MOSFET to ensure the full backscattering of electrons. The MOSFET device, including its 14-pin package, is mounted on a printed circuit board (PCB) at a depth of $70 \mathrm{~mm}$ below the surface of the PMMA phantom. A narrow channel is provided to allow cabling to connect the device to the measurement and irradiation equipment described above. In order to verify the dose delivered to the MOSFET device, a Farmer dosimeter with a chamber of volume of $0.6 \mathrm{~cm}^{3}$ (type NE 2571) was located at the same depth as the MOSFET device within the phantom. This chamber and related electrometer were calibrated according to national protocols and factors traced back to national dosimetry standards at the National Physical Laboratory, Teddington, Middlesex, UK. The Doseleader (type 2610) calibrated electrometer records the output of this chamber in order to ensure the consistency of the doses delivered.

In order to study RICN, a total of $12 p$-MOSFET devices were irradiated in two groups of six. All 12 devices were irradiated under a forward bias of $+5 \mathrm{~V}$ with all other device terminals grounded, which we shall henceforth term "primary" dose. One group of six were irradiated to a primary dose of $48 \mathrm{~Gy}$ and the other group to a primary dose of $80 \mathrm{~Gy}$. The threshold voltage of all MOSFETs was measured after this exposure. Each device was then irradiated in dose steps of $4 \mathrm{~Gy}$, with increasing steps in reverse bias. The threshold voltage was measured after each exposure as before. The devices were irradiated to photons produced from an Elekta linear accelerator (LINAC) operated at $6 \mathrm{MV}$ at the Clatterbridge Centre for Oncology National Health Service (NHS) Trust, Clatterbridge, UK.

\section{RESULTS}

Figure 3 is a plot of the change in threshold voltage $V_{t}$ in volts (V) versus dose in Gray (Gy) for a complete experimental cycle of this work. The change in threshold voltage is relative to zero dose, and implicitly we are considering a negative change, since radiation under positive bias leads to a reduction in threshold voltage in the $p$-MOS transistors used in this research. In Fig. 3 the behavior of the threshold voltage for the primary dose of $48 \mathrm{~Gy}$ is shown; similar trends were observed for the primary dose of 80 Gy and are not included in this work in the interests of brevity.

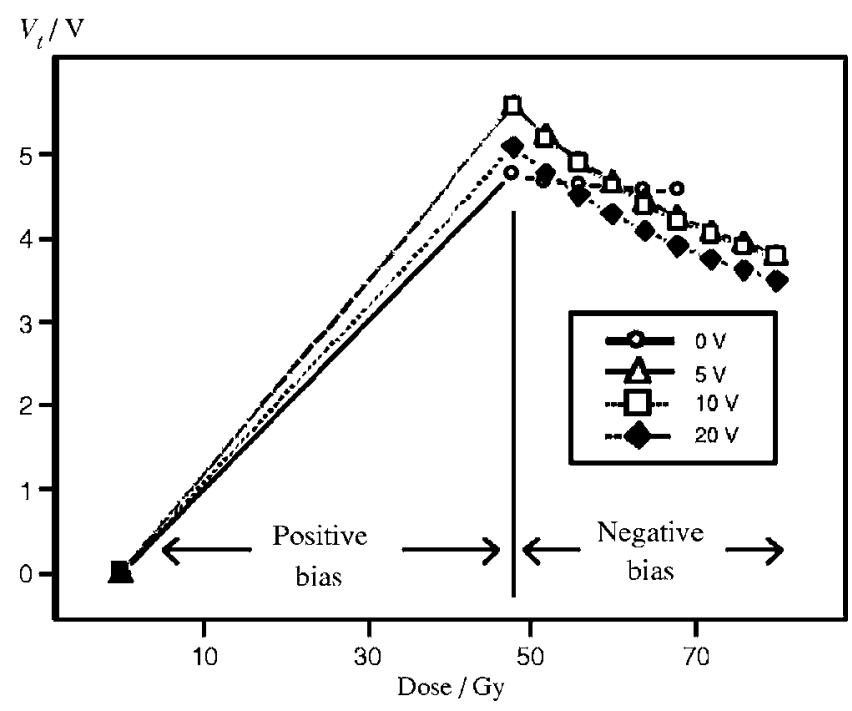

FIG. 3. Threshold voltage $V_{t}(\mathrm{~V})$ as a function of dose (Gy) for $p$-MOS transistors subject to positive bias and negative bias. Only those data for the transistors under reverse biases of $0,5,10$, and $20 \mathrm{~V}$ are shown for clarity. Initial dose under positive bias of $48 \mathrm{~Gy}$.

Under positive bias the threshold voltage changes significantly, following the well-known linear approximation that is valid for small dose levels. This dependence, borne out of an approximation to an exponential trend, is well documented elsewhere for the devices used in this work. For this reason, the data in between the extremes shown here have not been included. Beyond $48 \mathrm{~Gy}$, where the bias has been reversed, the threshold voltage is observed to fall gradually. Similar trends are observed for all levels of negative bias used.

Figures 4 and 5 show the data for threshold voltage change as a function of dose under negative bias for the primary doses of 48 and $80 \mathrm{~Gy}$, respectively. Nonlinear fits have been applied to each data set of the form

$$
V_{t}(D)=\alpha e^{-\beta D}
$$

where $\alpha$ and $\beta$ are constants and $D$ is dose. Note that the data in Figs. 4 and 5 have been normalized to zero $V_{t}$ at the point where the bias has been reversed. This removes any variation between the data sets for different devices and corresponds to a uniform value of $\alpha$ throughout the data. This has been done to extract the rate of $V_{t}$ recovery, which is described by $\beta$ in Eq. (1). The data for $\beta$, as a function of negative bias, are given in Table I.

In Fig. 6, the recovery parameter $\beta\left(\mathrm{Gy}^{-1}\right)$ has been plotted against negative bias (V), for the case of the two primary doses: 48 and $80 \mathrm{~Gy}$. The uncertainties in $\beta$ are smaller than the size of the symbols depicting the data. Two empirical fits have been applied to these data. These are

$$
\beta(V)=\frac{a}{V} \frac{1}{e^{b / V}-c} .
$$

The fit provided by this expression [Eq. (2)] has been optimized by raising the denominator in the exponent to a variable power $b$. This does not, however, reflect any further physical interpretation, 


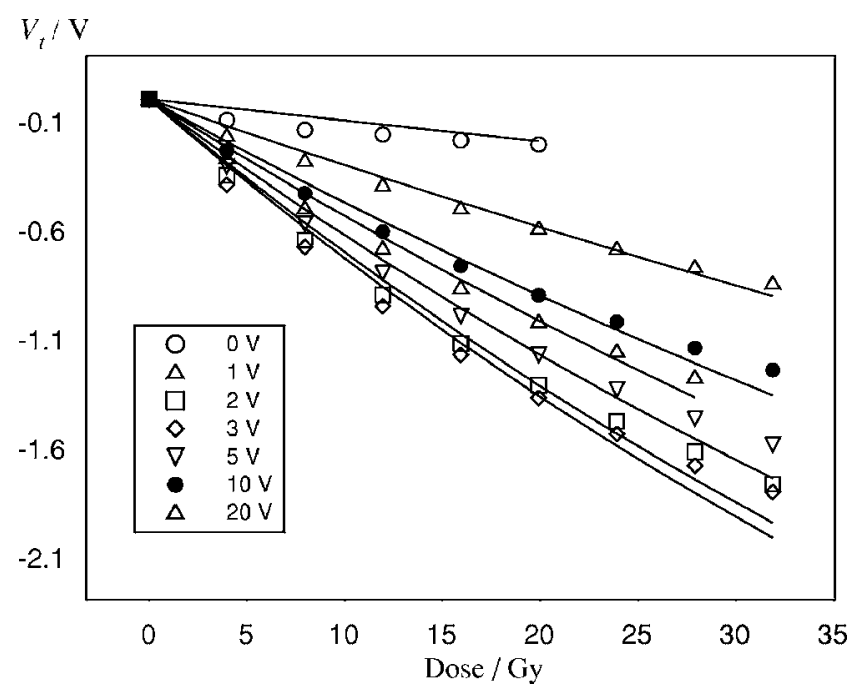

FIG. 4. Relative threshold voltage $\left(V_{t}\right)$ change vs dose (Gy) while under negative bias (data normalized to zero for first datum in all cases) together with nonlinear fits. Initial dose under positive bias of $48 \mathrm{~Gy}$.

$$
\beta(V)=\frac{a}{V} \frac{1}{e^{1 / V^{b}}-c},
$$

where $a, b$, and $c$ are constants for each case. Data for each of the fits listed above are given in Table II.

The degree of negative bias at which optimum RICN occurs corresponds to the bias at which $\beta$ is maximized. This has been determined via an iterative solution of the derivative of the model used to fit the dependence of $\beta$ with negative bias. This procedure has been carried out for Eqs. (2) and (3), thus obtaining solutions for $V$ such that

$$
b=V\left(1-c e^{-b / V}\right)
$$

in the case of Eq. (2) and

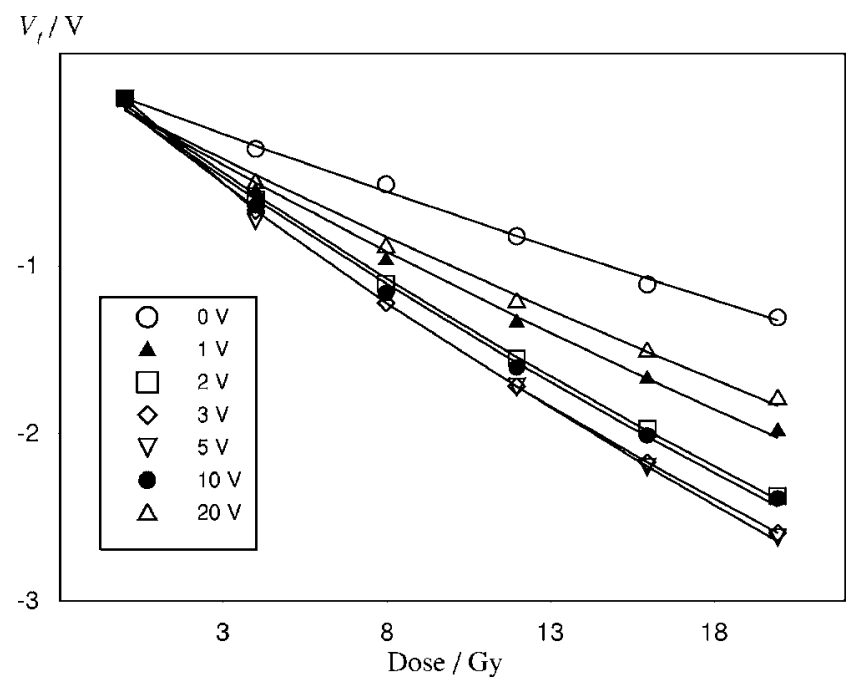

FIG. 5. Relative threshold voltage $\left(V_{t}\right)$ change vs dose (Gy) while under negative bias (data normalized to zero for first datum in all cases) together with nonlinear fits. Initial dose under positive bias of $80 \mathrm{~Gy}$.
TABLE I. RICN recovery parameter $\beta\left(\mathrm{kGy}^{-1}\right)$ as a function of negative bias (V) for primary doses of 48 and $80 \mathrm{~Gy}$.

\begin{tabular}{ccc}
\hline \hline & \multicolumn{2}{c}{$\beta\left(\mathrm{kGy}^{-1}\right)$} \\
\cline { 2 - 3 } Negative bias (V) & $48 \mathrm{~Gy}$ & $80 \mathrm{~Gy}$ \\
\hline 0 & $2.10 \pm 0.02$ & $8.40 \pm 0.08$ \\
1 & $9.60 \pm 0.09$ & $12.6 \pm 0.1$ \\
2 & $13.6 \pm 0.1$ & $16.2 \pm 0.2$ \\
3 & $14.2 \pm 0.1$ & $17.6 \pm 0.2$ \\
4 & $\cdots$ & $18.2 \pm 0.2$ \\
5 & $13.2 \pm 0.1$ & $17.7 \pm 0.2$ \\
10 & $10.20 \pm 0.09$ & $16.0 \pm 0.1$ \\
20 & $6.00 \pm 0.05$ & $11.4 \pm 0.1$ \\
\hline \hline
\end{tabular}

$$
b=V^{b}\left(1-c e^{-1 / V^{b}}\right)
$$

for Eq. (3). These results are given in Table III for each of the primary doses considered in this research, together with the optimum value of $\beta$ in each case.

\section{DISCUSSION}

\section{Primary dose dependence and an optimum RICN level}

The optimum bias level is observed within uncertainties in the data of $2 \%$ and consistently by both expressions (2) and (3). The data arising from the two primary doses investigated in this work indicate that the level of primary dose does not appear to influence the bias at which optimum RICN occurs. However, this is clearly only based on two measurements so this interpretation must be treated with caution.

The levels of negative bias corresponding to optimum RICN, measured in this work, are consistent with the range in negative bias for optimum RICN rate reported in previous studies. ${ }^{19}$ However, an approximately constant dependence with reverse bias, as has been suggested, is not observed. The previous studies were of devices with much thinner oxides, putting the corresponding oxide field regimes in different classes and thus increasing the influence of interface

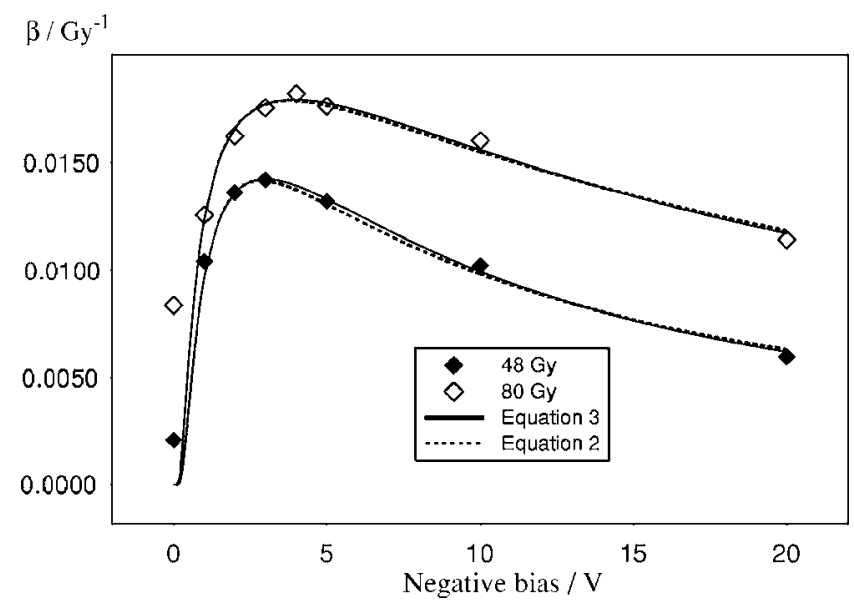

FIG. 6. Recovery parameter $\beta\left(\mathrm{Gy}^{-1}\right)$ against negative gate bias (V) for primary doses of 48 and 80 Gy. Fits are included for Eqs. (2) and (3). 
TABLE II. Fit parameters for various models to describe the variation of RICN recovery parameter $\beta$ with negative bias.

\begin{tabular}{|c|c|c|c|c|c|c|c|c|}
\hline \multirow[b]{2}{*}{ Model } & \multicolumn{4}{|c|}{ Coefficients of fits (primary dose of $48 \mathrm{~Gy}$ ) } & \multicolumn{4}{|c|}{ Coefficients of fits (primary dose of $80 \mathrm{~Gy}$ ) } \\
\hline & $a\left(\mathrm{~V} \mathrm{~Gy}^{-1}\right)$ & $b(\mathrm{~V})$ & $c$ & $\chi_{\nu}^{2}$ & $a(\mathrm{~V})$ & $b(\mathrm{~V})$ & $c$ & $\chi_{\nu}^{2}$ \\
\hline Equation (3) & 0.018 & 1.231 & 0.883 & 1.746 & 0.022 & 1.081 & 0.947 & 1.415 \\
\hline Equation (2) & 0.040 & 1.607 & 0.767 & 2.719 & 0.030 & 1.216 & 0.936 & 1.740 \\
\hline
\end{tabular}

states significantly. Furthermore, much larger doses were used in comparison with this research. By contrast, the data in this work exhibit a nonlinear dependence on either side of the optimum bias level.

\section{The dependence of recovery with reverse bias}

The two fits to the dependence of the recovery parameter $\beta$ with reverse bias, shown in Fig. 6, are both acceptable statistical analyses of the RICN data. Expression (3) gives the best representation of the data for both primary doses. If a deeper physical interpretation of RICN data is not required then this is the best model to use. The usual caveat associated with radiation effects in MOS transistors should be borne in mind, i.e., that devices from contrasting fabrication sources will exhibit statistical variation around and possibly beyond the fit parameters given here. However, it is reasonable to expect the trends across MOSFET dosimeters from different manufacturers to be similar. The dependence of RICN with reverse bias observed in this research agrees qualitatively with the hypothesis ${ }^{19}$ that, at high levels of negative bias, the electric field in the oxide transports the electrons past the trapped holes and thus RICN is limited in comparison with that at lower levels of reverse bias. RICN is observed to increase until the optimum because the processes of compensation and recombination are encouraged as electrons are transported less rapidly, due to the falling electric field. Below the optimum, however, electron transport appears to be incomplete as we approach the positive bias regime; thus RICN falls with further reductions in reverse bias below the optimum, until it eventually ceases completely at the extreme where transport is no longer reversed.

\section{The correspondence of $\beta$ with RICN cross section}

The statistics of electron trapping are often considered by equating the rate of electron trapping with that of detrapping via photon and phonon absorptions. In the case of low electric fields, i.e., $<1.5 \times 10^{6} \mathrm{~V} \mathrm{~cm}^{-1}$, the cross sections for these detrapping mechanisms are small. Raising the electric

TABLE III. Negative bias values for optimum levels of RICN recovery parameter $\beta\left(\mathrm{kGy}^{-1}\right)$, for primary doses of 48 and $80 \mathrm{~Gy}$, arising from Eqs. (2) and (3).

\begin{tabular}{cccc}
\hline \hline \multirow{2}{*}{$\begin{array}{c}\text { Model } \\
\text { Primary absorbed dose }\end{array}$} & \multicolumn{2}{c}{ Negative bias (V) } & \\
\cline { 2 - 3 } & $48 \mathrm{~Gy}$ & $80 \mathrm{~Gy}$ & Optimum $\beta\left(\mathrm{kGy}^{-1}\right)$ \\
\hline Equation (2) & $2.85 \pm 0.05$ & $2.85 \pm 0.03$ & $14.2 \pm 0.1$ \\
Equation (3) & $3.00 \pm 0.03$ & $3.00 \pm 0.03$ & $16.2 \pm 0.1$ \\
\hline \hline
\end{tabular}

field reduces the energy depth of the trap center which raises the significance of impact ionization and field ionization in detrapping. These influences are often folded into the field dependence of the trapping cross section. ${ }^{23}$ In addition to Coulombic traps, which acquire charge carriers via traditional charge-based attraction, neutral traps can arise from strained molecular bonds in the oxide's atomic lattice. The cross section of these is likely to have a less-pronounced field dependence because of the much shorter effective range of the potential of the neutral trap. ${ }^{24}$

In this work the maximum oxide electric field $E_{\text {ox }}$ was $5 \times 10^{5} \mathrm{~V} \mathrm{~cm}^{-1}$. Electron capture by Coulomb-attractive oxide-charge centers, leading to recombination, is known to be a strong function of the average oxide field. ${ }^{25}$ For $E_{\text {ox }}$ $<10^{6} \mathrm{~V} \mathrm{~cm}^{-1}$ (this case) the cross section for recombination $\sigma_{r}$ is known to vary approximately as $E_{\mathrm{ox}}^{-3 / 2}$. The data beyond the point of optimum RICN (for negative bias levels greater than $3 \mathrm{~V}$ ) do not exhibit even an approximate correspondence with this dependence. Instead a much reduced dependence of approximately $E_{\mathrm{ox}}^{-1 / 3}$ is observed. Clearly, a significant shortcoming of our work is that in this region there are very few data. However, the inconsistency of the field dependence may support the possibility of a combined role of recombination and compensation in RICN, as opposed to that of recombination alone. Alternatively, the neutralization phenomenon may be due to the localized trapping of electrons in neutral traps, thus exhibiting the less pronounced field dependence described above.

Returning to Eq. (2) and considering RICN in terms of recombination cross section $\sigma_{r}$, we can draw correspondence with the known dependence of this ${ }^{25}$ with threshold voltage change $V_{t}$ :

$$
V_{t}=\frac{q N_{\mathrm{TT}} \bar{x}}{K_{\mathrm{ox}} \epsilon_{0}}\left[1-e^{-\sigma_{r} N_{\mathrm{inj}}}\right] .
$$

Here $N_{\mathrm{TT}}$ is the number of trap centers per unit area, $\bar{x}$ is the centroid of the trap distribution, $K_{\mathrm{ox}}$ is the static dielectric constant of $\mathrm{SiO}_{2}, N_{\text {inj }}$ is the number of electrons introduced per unit area, $q$ is the charge of an electron, and $\epsilon_{0}$ is the permittivity of a vacuum. If $V_{t}$ in Eq. (1) is considered as a saturating quantity, i.e., $V_{t}=\alpha\left(1-e^{-\beta D}\right)$, for comparison with Eq. (6) where $\beta$ is identical to that defined in Eq. (1), the following proportionality can be inferred:

$$
\sigma_{N} N_{\text {inj }} \propto \beta D .
$$

Here correspondence is drawn with radiation dose being the source of carriers as opposed to hot electron injection techniques. The latter have often been used to introduce carriers in experiments to measure the field dependence of trapping 
cross sections. Therefore $\sigma_{N}$ has been introduced in Eq. (7) as the cross section for RICN, to reflect the combined role of compensation and recombination of electrons and holes in the oxide. Since it has been shown that $\beta$ is a function of oxide field, it follows that $\sigma_{N}$ will also exhibit a related dependence, i.e., $\sigma\left(E_{\mathrm{ox}}\right)$ and $\beta\left(E_{\mathrm{ox}}\right)$,

$$
\sigma_{N}\left(E_{\mathrm{ox}}\right) \propto \frac{\beta\left(E_{\mathrm{ox}}\right) D}{N_{\mathrm{inj}}} .
$$

Thus the experimental measurement of the dependence of $\beta$ with reverse bias provides a qualitative means for characterizing the dependence of cross section for RICN with electric field across the oxide.

\section{The implications for dose measurement}

RICN is unlikely to be of use in mainstream dosimetry with MOS devices. However, in extreme circumstances where switch-bias conditions arise, either inadvertently or otherwise, the dependence of the neutralization of trapped charge with reverse bias will be crucial if adjustments are sought to account for the effect of the exposure under reverse bias. This may, for example, be of particular value where MOS devices are inaccessible and cannot be replaced easily, such as in space. Furthermore, the possible independence of optimum RICN bias with primary dose suggests that allowance for switch-bias effects could be made with reference to this optimum recovery level and the dependence of $\beta$ with bias reported in this research.

\section{CONCLUSIONS}

The dependence of RICN with reverse bias has been measured for doses consistent with therapeutic uses of these devices, up to $20 \mathrm{~Gy}$. Correspondence between the dependence of RICN with reverse bias and the dependence of neutralization cross section with oxide electric field has been made. In summary, this work can be concluded as follows.

(1) For high levels of negative bias, the degree of RICN falls off to very low levels, consistent with the works of Fleetwood $^{19}$ and of Freitag et al. ${ }^{26}$

(2) In the high-level, reverse bias extreme, the degree of RICN is very low but it is observed to be greater than that for very low levels of reverse bias, i.e., for bias levels greater than $-1 \mathrm{~V}$.

(3) The dependence of RICN between -1 and $-6 \mathrm{~V}$ is observed to be nonlinear and a maximum is observed in this region.

(4) The reverse bias level at the optimum RICN is consistent with the region identified previously, ${ }^{19}$ corresponding to the region in which the gradient of the threshold voltage change versus reverse bias is maximized.

(5) The observation that an optimum bias exists for RICN suggests that it is at this point that the field due to the oxide trap charge becomes greater than the applied gate bias. If this is the case, then charge transport at this optimum level must be small, due to the fields essentially canceling each other. Hence this provides further evidence for the location of the $\mathrm{H}^{+}$ions responsible for making the interface traps being very near the $\mathrm{Si} / \mathrm{SiO}_{2}$ interface.

(6) A proportional relationship exists between the crosssection dependence of RICN with oxide field and that of the experimental recovery parameter $\beta$ observed as a result of RICN measurements.

(7) The dependence of RICN versus reverse bias follows an empirical dependence consistent with Eq. (2). More research is necessary to determine how primary dose influences the reverse bias level associated with optimal RICN.

\section{ACKNOWLEDGMENTS}

We gratefully acknowledge the support and assistance of the Engineering and Physical Sciences Research Council (EPSRC), the Clatterbridge Centre for Oncology, and the General Organization for Technical Education and Vocational Training, Riyadh, Saudi Arabia.

${ }^{1}$ L. S. August, IEEE Trans. Nucl. Sci. 29, 2000 (1982).

${ }^{2}$ A. Holmes-Siedle, Nucl. Instrum. Methods 121, 169 (1974).

${ }^{3}$ A. Jones and D. Hintenlang, Health Phys. 82, S130 (2002).

${ }^{4}$ A. B. Rosenfeld, Radiat. Prot. Dosim. 101, 393 (2002).

${ }^{5}$ J. R. Schwank, S. B. Roeske, and D. E. Beutler, IEEE Trans. Nucl. Sci. 43, 2671 (1996).

${ }^{6}$ M. W. Bower and D. E. Hintenlang, Health Phys. 75, 197 (1998).

${ }^{7}$ M. J. Butson, A. Rosenfeld, J. N. Mathur, M. Carolan, T. P. Wong, and P. E. Metcalfe, Med. Phys. 23, 655 (1996).

${ }^{8}$ K. Y. Quach, J. Morales, M. J. Butson, A. B. Rosenfeld, and P. E. Metcalfe, Med. Phys. 27, 1676 (2000).

${ }^{9}$ R. Ramani, S. Russell, and P. O'Brien, Int. J. Radiat. Oncol., Biol., Phys. 37, 959 (1997).

${ }^{10}$ S. L. Dong, T. C. Chu, J. S. Lee, G. Y. Lan, T. H. Wu, Y. H. Yeh, and J. J. Hwang, Appl. Radiat. Isot. 57, 791 (2002).

${ }^{11}$ S. L. Dong, T. C. Chu, G. Y. Lan, T. H. Wu, Y. C. Lin, and J. S. Lee, Appl. Radiat. Isot. 57, 883 (2002).

${ }^{12}$ T. Kron, A. Rosenfeld, M. Lerch, and S. Bazley, Radiat. Prot. Dosim. 101, 445 (2002).

${ }^{13}$ C. F. Chuang, L. J. Verhey, and X. Ping, Med. Phys. 29, 1109 (2002).

${ }^{14}$ J. N. Roshau and D. E. Hintenlang, Health Phys. 84, 376 (2003).

${ }^{15}$ B. D. Pomije, C. H. Huh, M. A. Tressler, D. E. Hintenlang, and W. E. Bolch, Health Phys. 80, 497 (2001).

${ }^{16}$ R. A. Price, C. Benson, M. J. Joyce, D. J. Kestell, and J. Silvie, Radiat. Prot. Dosim. 110, 283 (2004).

${ }^{17}$ C. Benson, R. A. Price, J. Silvie, A. Jaksic, and M. J. Joyce, Phys. Med. Biol. 49, 3145 (2004).

${ }^{18}$ R. A. Price, C. Benson, M. J. Joyce, and K. Rodgers, IEEE Trans. Nucl. Sci. 51, 1420 (2004).

${ }^{19}$ D. M. Fleetwood, J. Appl. Phys. 67, 580 (1990).

${ }^{20}$ Y. P. Tsvidis, Operation and modelling of the MOS transistor (Oxford University Press, New York, 2003).

${ }^{21}$ D. M. Fleetwood, IEEE Trans. Nucl. Sci. 39, 269 (1992).

${ }^{22}$ Z. Savic, B. Radjenovic, M. Pejovic, and N. Stojadinovic, IEEE Trans. Nucl. Sci. 42, 1445 (1995).

${ }^{23}$ D. A. Buchanan, M. V. Fischetti, and D. J. DiMaria, Phys. Rev. B 43, 1471 (1991).

${ }^{24}$ S. K. Lai, J. Appl. Phys. 54, 2540 (1983).

${ }^{25}$ T. H. Ning, J. Appl. Phys. 47, 3203 (1976).

${ }^{26}$ R. K. Freitag, C. M. Dozier, and D. B. Brown, IEEE Trans. Nucl. Sci. NS-34, 1172 (1987) 